\title{
Acute pituitary apoplexy complicating a pituitary macroadenoma
}

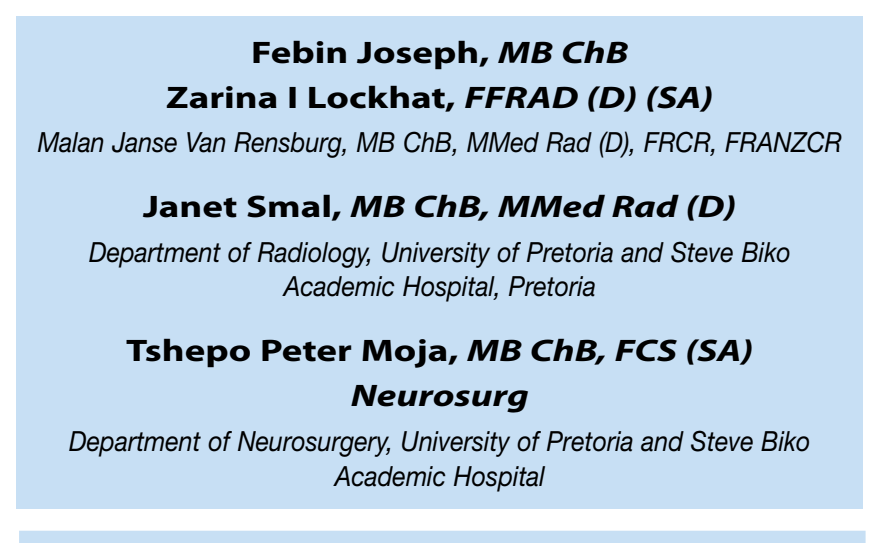

\section{Abstract}

Pituitary apoplexy is a rare but potentially life-threatening condition caused by either haemorrhage or infarction of the pituitary gland. In most cases, a pre-existing pituitary macroadenoma is present. Patients present with the clinical syndrome of headache, visual defects or ophthalmoplegia, altered state of consciousness and variable endocrine deficits.

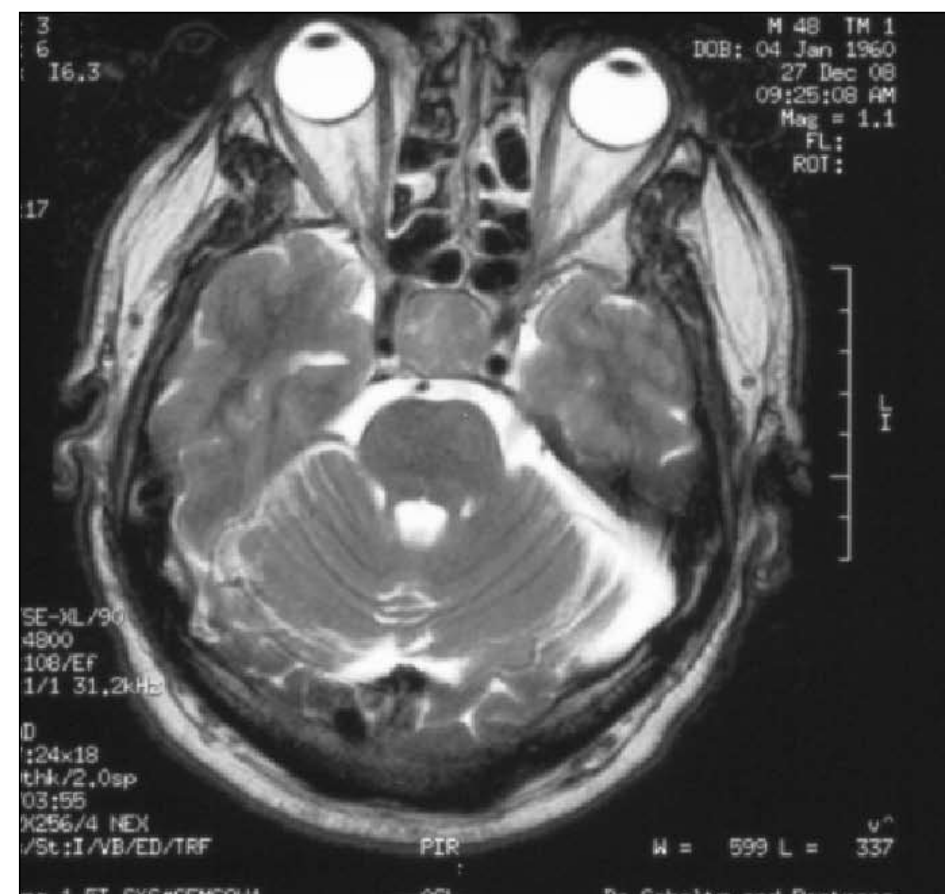

\section{Case history}

A 49-year-old man presented to the emergency department with headaches, visual disturbances and severe hypotension. On examination, a left temporal hemianopia was found. His GCS was 15/15 and there were no other cranial nerve deficits. Laboratory investigations revealed decreased cortisol, thyroid stimulating hormone, prolactin, testosterone and adreno-cortico-thyrotropic hormone levels. He was also hyponatraemic.

A clinical diagnosis of pituitary apoplexy with severe hypopituitarism was made, and the patient had immediate fluid and electrolyte replacement, hydrocortisone and T4 replacement. The patient was reluctant to undergo surgery and was initially managed with medical therapy.

\section{Imaging findings}

On MR investigation, a sella and supra-sella mass showing mixed signal intensity was found (Figs 1 - 3). Subsequent imaging to plan for transsphenoidal surgery included a CT scan of the skull base and brain, done 3 months after the initial diagnosis, and it was then discovered that the sella mass had decreased in size. Further MR imaging a week after the CT scan and again 2 months later (Figs 4 - 6) revealed a

\footnotetext{
Fig. 1. Axial T2W and T2 FLAIR images demonstrate a mass of heterogeneous signal intensity within the pituitary fossa.
} 


\section{CASE REPORT}

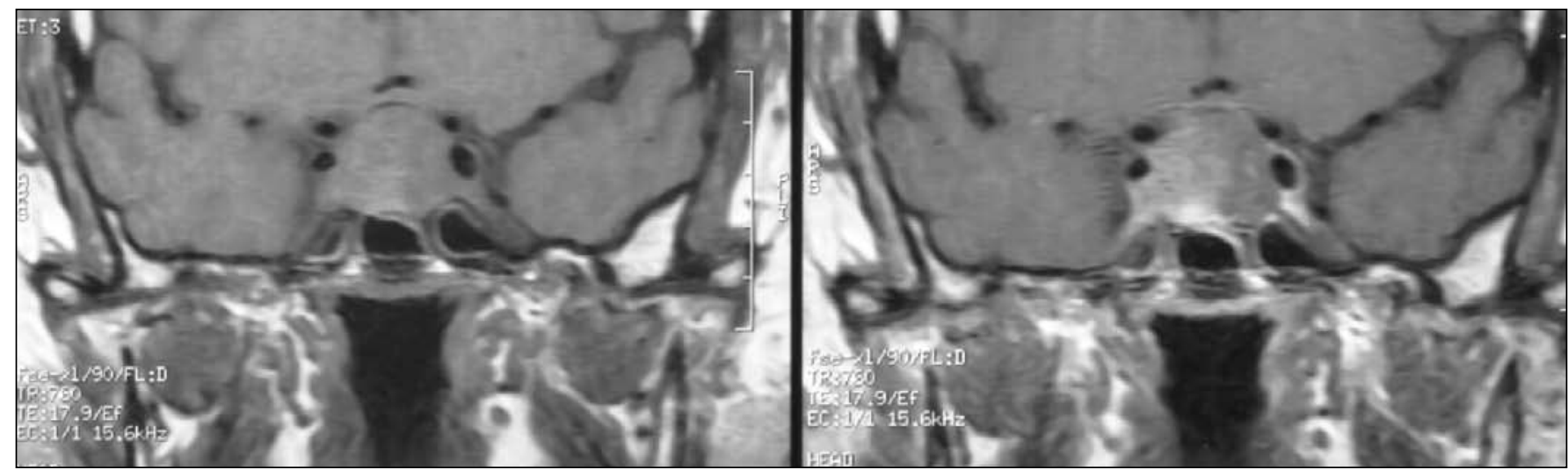

Fig.2. Coronal TIW images, pre- and post-gadolinium, demonstrate expansion of the pituitary mass with resultant displacement and compression of the optic chiasm superiorly and displacement of the internal carotid arteries and cavernous sinuses laterally. Inhomogeneous contrast enhancement is evident.

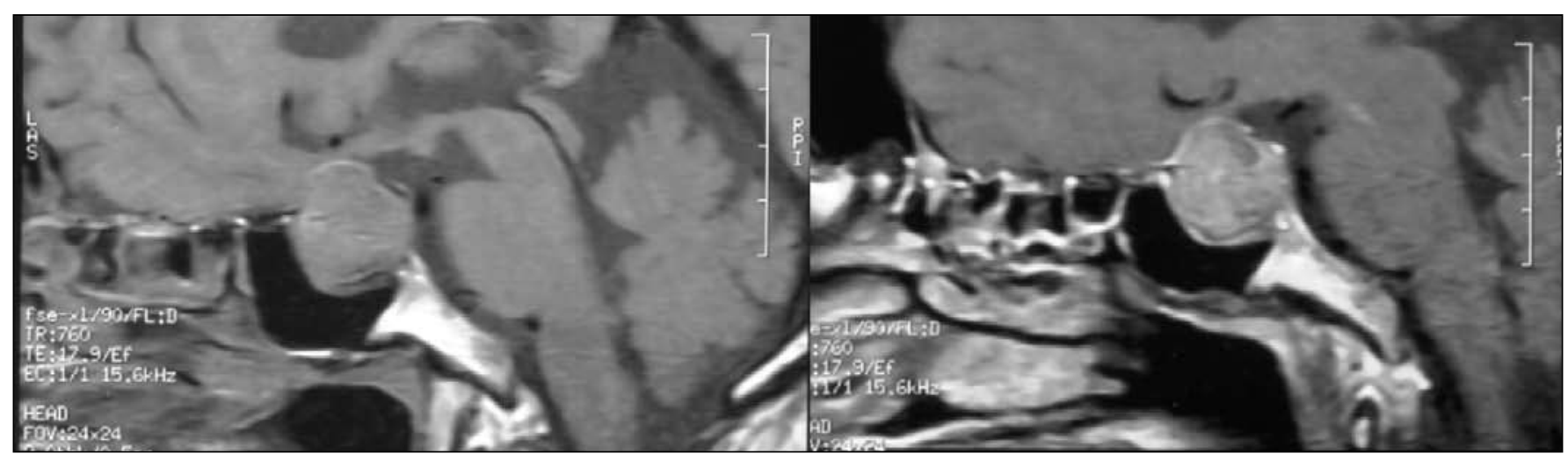

Fig. 3. Sagittal TIW images, pre- and post-gadolinium, show expansion of the pituitary fossa by the pituitary mass. The suprasellar cistern is obliterated. Inhomogeneous contrast enhancement of the mass is also evident.

further reduction in tumour volume. A final diagnosis of acute pituitary apoplexy complicating a pituitary macro-adenoma was made.

\section{Discussion}

The first case of a pituitary tumour associated with haemorrhage was described by Bailey in 1898. ${ }^{1}$ The signs and symptoms of pituitary tumour infarction or haemorrhage are collectively termed 'pituitary apoplexy', which term was first used by Brougham et al. ${ }^{2}$ Verrees et al. further expanded this term to 'pituitary tumour apoplexy' because of the overwhelming number of cases associated with pituitary adenoma and only isolated instances of apoplexy in healthy glands. ${ }^{3}$

Pituitary apoplexy occurs in approximately $1 \%$ of macro-adenomas; in 65 - 95\% of cases, a pre-existing macro-adenoma is present. ${ }^{4}$ Commonly, the episode of apoplexy occurs spontaneously; however, several risk factors have been implicated in the development of apoplexy. These include anticoagulation, dynamic pituitary function tests, radiation, bromocriptine therapy for existing macro-adenoma, trauma, surgery, peri- or post-partum state, elevated oestrogen levels and diabetes. ${ }^{4}$

Up to $20 \%$ of pituitary apoplexy cases may be sub-clinical, but with radiological and pathological evidence of a haemorrhagic infarction.

The clinical signs and symptoms are a result of the expanding pituitary mass secondary to haemorrhage or non-haemorrhagic infarction,

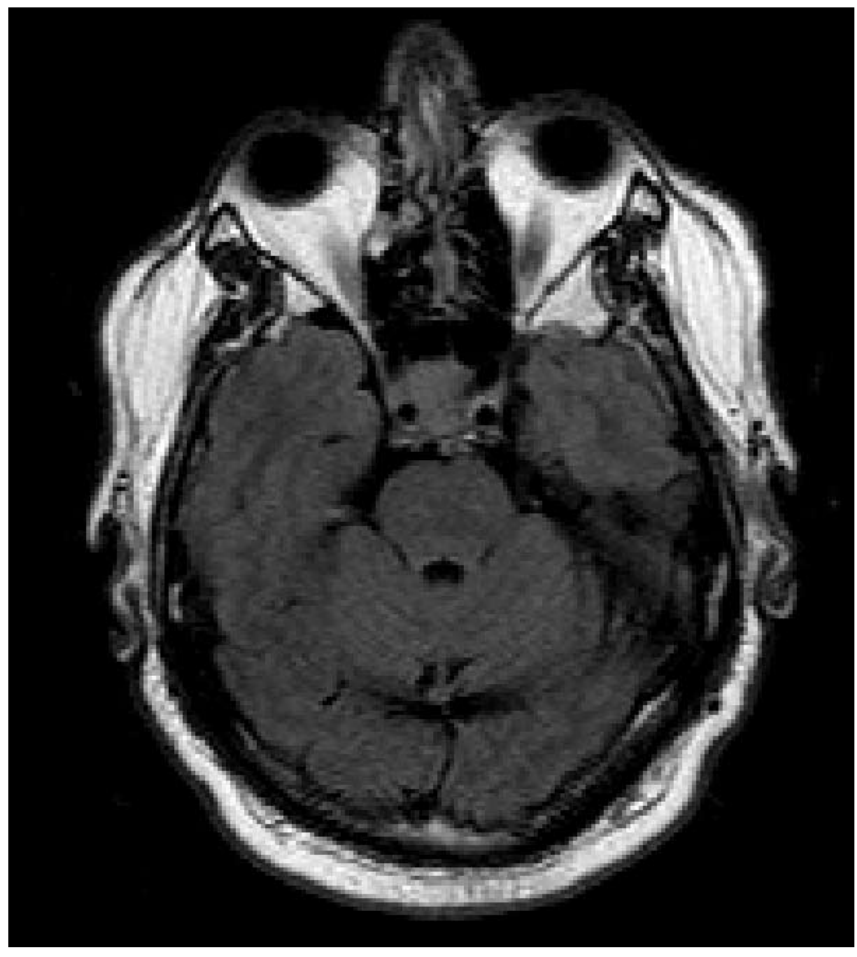

Fig. 4. Axial T2 FLAIR image shows reduction in tumour volume 


\section{CASE REPORT}

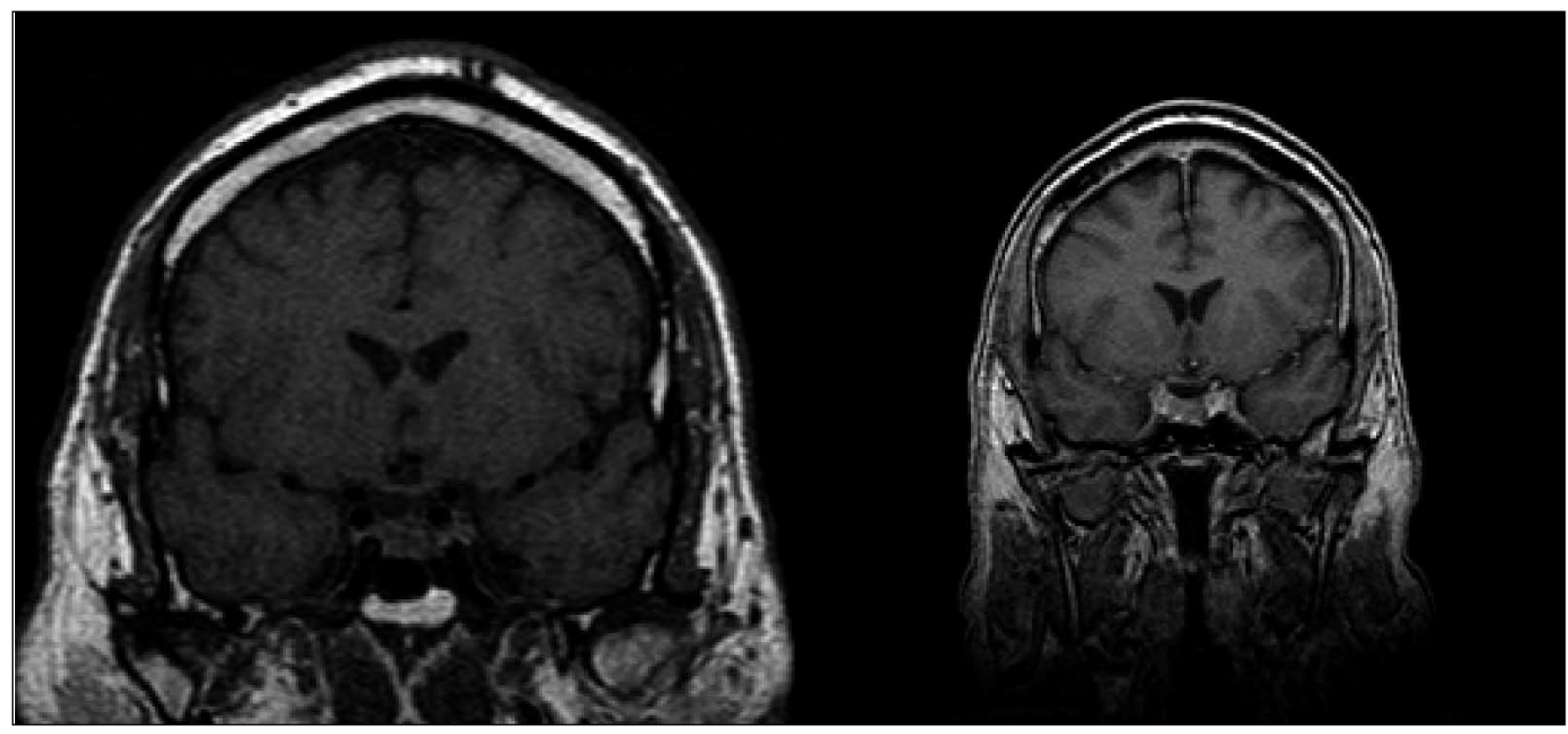

Fig. 5. Coronal T1W images pre and post gadolinium show a smaller pituitary mass. The mass effect that was previously exerted on the optic chiasm and cavernous sinuses is no longer evident.

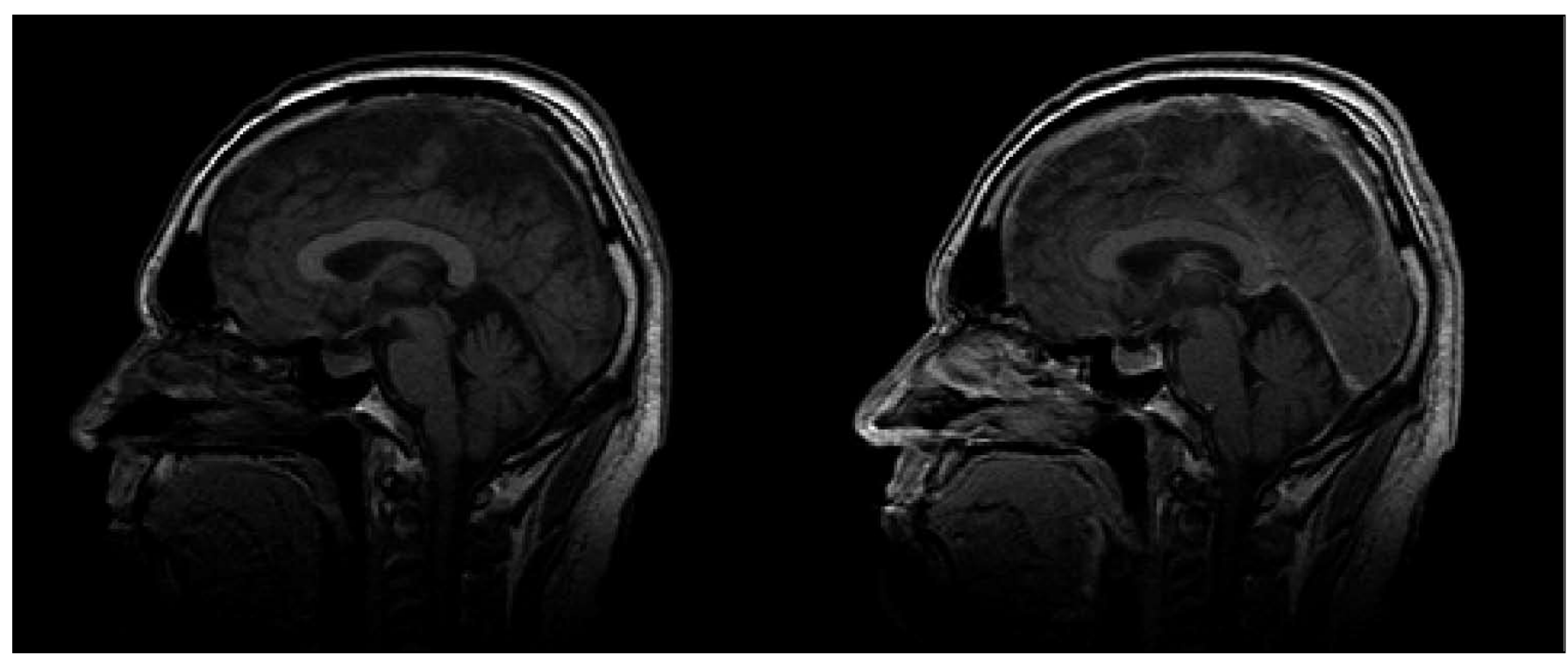

Fig.6. Sagittal T1W images pre and post gadolinium, now demonstrating a smaller mass.The suprasellar cistern is now visible.

with resultant compression of the adjacent structures, viz. cavernous sinus and neuro-vascular bundle (hence cranial nerve palsies and ophthalmoplegia). ${ }^{5}$ Headache occurs secondary to meningeal irritation owing to blood entering the basal cisterns or from dural stretching. Blood in the supra-sellar cistern may provoke chemical meningitis, as has been reported by Wen-Yi et al. ${ }^{6}$

Restricted visual fields are the result of distension of the optic nerves and chiasm, specifically bitemporal hemi-anopia. Lateral haemorrhage and necrosis leads to deficits in the 3rd, 4th, 5th (1st and 2nd branches) and 6th cranial nerves.
A decreased level of consciousness may be the result of endocrine abnormalities associated with the hypopituitarism or pressure transmitted to the brainstem or owing to hypothalamic compression. ${ }^{3}$ A life-threatening pituitary insufficiency or an acute adrenal crisis may occur. $^{4}$

Most of the patients who present with apoplexy have a hypopituitarism with decreased levels of all or several pituitary hormones. Three causes for this have been postulated that include elevated intra-sellar pressure compressing the pituitary, pre-existing deficits from the adenoma itself, and destruction of the pituitary gland. ${ }^{3}$ Diabetes insipidus is rarely a 


\section{CASE REPORT}

feature because the posterior lobe is usually not part of the adenoma.

The imaging findings on contrast-enhanced CT reveal a pituitary mass with peripheral enhancement, with or without haemorrhage. Patchy or confluent hyperdensity may be seen on non-enhanced CT. An associated sub-arachnoid haemorrhage can sometimes be present.

On T1W MR imaging, in the early acute stage, an enlarged gland that is iso- or hypointense to brain can be seen, while in the late acute or sub-acute stage, a hyperintense gland is seen. Acute compression of the hypothalamus and optic chiasm may cause hyperintensity along the optic tracts on T2W MR images. In the chronic stage, a hyperintense 'empty' sella filled with CSF can be demonstrated on T2W images. Restricted diffusion within an adenoma may be an early sign of apoplexy. Contrast administration often reveals rim-enhancement.

The differential diagnoses to consider include a non-haemorrhagic pituitary macro-adenoma, a craniopharyngioma, a Rathke cleft cyst, a pituitary abscess, primary intra-pituitary haemorrhage, or a giant thrombosed intra-sellar aneurysm.

Pituitary apoplexy is a neurosurgical emergency that requires urgent surgical decompression to prevent permanent visual loss and possible death from local pressure on the hypothalamus and brainstem. The pituitary gland remains capable of secreting adequate amounts of hormones when as little as $10 \%$ of residual tissue remains. ${ }^{3}$ Prompt diagnosis allows the initiation of steroid, fluid and electrolyte replacement; definitive treatment by surgical decompression is then required. ${ }^{3,4}$

\section{Conclusion}

Pituitary apoplexy should be considered in a patient who presents with a sudden onset of headache, visual field abnormalities and ocular muscle paresis. MRI is the most sensitive diagnostic imaging modality, and prompt diagnosis expedites immediate treatment.

1. Bailey P. Pathological report of a case of acromegaly, with especial reference to the hypophysis cerebri and in the thyroid gland; and a case of haemorhage into the pituitary. Philadelphia Med J 1898;1:789.

2. Brougham M, Heusner AP, Adams RD. Acute degenerative changes in adenomas of the pituitary bodywith special reference to pituitary apoplexy. J Neurosurg 1950;7:421.

3. Verrees M, Arafah BM, Selman WR. Pituitary tumour apoplexy: characteristics, treatment, and outcomes. Neurosurgical Focus 2004. http://www.medscape.com/viewarticle/474903_6 (accessed 3 August 2009).

4. Osborn AG. Diagnostic Imaging: Brain, Sella and Pituitary, 1st ed. Salt Lake City, USA: Amirsys, 2004:II2:28-30.

5. Goel V, Debnath UK, Singh J, Brydon H. Pituitary apoplexy after joint arthroplasty. J Arthroplasty 2009; 24(5):826.e7-826.e10.

6. Wen-Yi H, Chien $\mathrm{Y}, \mathrm{Wu}$ C, et al. Pituitary adenoma apoplexy with initial presentation mimicking bacterial meningoencephalitis: a case report. Am J Emerg Med 2009;27: 517.el-517.e4. 